\title{
New anisotropic models from isotropic solutions
}

\author{
S. D. Maharaj* and M. Chaisi ${ }^{\dagger}$ \\ Astrophysics and Cosmology Research Unit \\ School of Mathematical Sciences \\ University of KwaZulu-Natal \\ Durban 4041, South Africa
}

\begin{abstract}
We establish an algorithm that produces a new solution to the Einstein field equations, with an anisotropic matter distribution, from a given seed isotropic solution. The new solution is expressed in terms of integrals of known functions, and the integration can be completed in principle. The applicability of this technique is demonstrated by generating anisotropic isothermal spheres and anisotropic constant density Schwarzschild spheres. Both of these solutions are expressed in closed form in terms of elementary functions, and this facilitates physical analysis.
\end{abstract}

AMS Classification numbers: 83C15; 85A99

*Author for correspondence; email: maharaj@ukzn.ac.za; fax: +27312602632

${ }^{\dagger}$ Permanent address: Department of Mathematics \& Computer Science, National University of Lesotho, Roma 180, Lesotho; eMail: m. chaisi@nul.ls 


\section{Introduction}

Solutions to the Einstein field equations with anisotropic matter, where the radial component of the pressure is not the same as the tangential component, have been studied by a variety of investigators in recent years. Such solutions are important in physics as they may be used to model anisotropic stars where a static spherically symmetric interior is matched to the Schwarzschild exterior model. These include the treatments of Dev and Gleiser [1, 2], Herrera et al. 3, 4], Ivanov [5], Mak and Harko [6, 7] and Sharma and Mukherjee 8]. As pointed out in these analyses anisotropy cannot be neglected when studying the critical mass and redshift of highly compact bodies, and may be an intrinsic component of boson stars and strange stars.

In generating exact models describing anisotropic matter distributions ad hoc assumptions are normally made about the forms of the matter variables or gravitational potentials. In an attempt to systematically generate exact solutions Maharaj and Maartens 9] proposed an algorithm in which the field equations are written as a first order system of differential equations. The energy density and radial pressure are then chosen on physical grounds; the remaining variables then follow from the field equations. The Maharaj and Maartens algorithm has been used by Gokhroo and Mehra [10], Chaisi and Maharaj [11, 12] amongst others to produce physically reasonable anisotropic stars. However these models have the feature that the anisotropy factor is nonzero in the interior of the star and an isotropic solution cannot be regained. The objective of this paper is to provide a new algorithm that may be used to systematically produce anisotropic solutions to the Einstein field equations from a given isotropic solution. This solution generating algorithm 
has the desirable property of yielding a new exact anisotropic model which includes an isotropic solution, unlike the earlier algorithm of Maharaj and Maartens. The new solutions obtained are members of a one parameter family containing the isotropic case. We illustrate the utility of the algorithm with two examples that are physically relevant, the isothermal sphere and the constant density Schwarzschild sphere.

The principal objective of this paper is to show that we can generate anisotropic relativistic stars from a specified seed isotropic star. In Section 2, we describe the basic features of the model for a relativistic anisotropic star, and present the differential equations, as a first order system, governing the gravitational field. The algorithm that produces a new anisotropic solution from a seed isotropic solution is fully derived in Section 3, and we present the final result in the form of a theorem. The isotropic isothermal model is used to generate a new anisotropic solution in Section 4, to illustrate the theorem. The conventional isothermal solution with a linear equation of state is contained in the new class of models. As a second example the interior Schwarzschild model is used to generate a new anisotropic solution in Section 5. The conventional interior Schwarzschild solution with constant density is contained in the new class of models. The details of the integration process for the second example is given in the Appendix for convenience. For both examples we study in brief the profile of the anisotropy factor. In Section 6 , we briefly indicate that the solutions found can be related to stellar models used in astronomy. 


\section{$2 \quad$ Field equations}

We utilise a representation of the field equations in which only first order derivatives appear. This helps to simplify the integration process as pointed out by Chaisi and Maharaj [1] whose notation and conventions we follow. We take the line element for static spherically symmetric spacetimes to be

$$
\mathrm{d} s^{2}=-e^{\nu} \mathrm{d} t^{2}+e^{\lambda} \mathrm{d} r^{2}+r^{2}\left(\mathrm{~d} \theta^{2}+\sin ^{2} \theta \mathrm{d} \phi^{2}\right)
$$

where $\nu(r)$ and $\lambda(r)$ are arbitrary functions. The energy-momentum tensor for isotropic matter which is not radiating has the form

$$
T^{a b}=(\mu+p) u^{a} u^{b}+p g^{a b}
$$

where the energy density $\mu$, and the isotropic pressure $p$ are measured relative to the four-velocity $u^{a}=e^{-\nu / 2} \delta_{0}^{a}$ which is comoving. We define the mass function as

$$
m(r)=\frac{1}{2} \int_{0}^{r} x^{2} \mu(x) \mathrm{d} x
$$

so that $M=m(R)$ is the total mass of a sphere of radius $R$. The Einstein field equations, with the help of (11)-(3) are equivalent to the system

$$
\begin{aligned}
e^{-\lambda} & =1-\frac{2 m}{r} \\
r(r-2 m) \nu^{\prime} & =p r^{3}+2 m \\
(\mu+p) \nu^{\prime}+2 p^{\prime} & =0
\end{aligned}
$$

for isotropic matter distributions. We are using units where the speed of light $(c)$ and the coupling constant $\left(8 \pi G / c^{4}\right)$ are unity.

The energy-momentum tensor for anisotropic matter which is not radiating has the form

$$
T^{a b}=(\mu+p) u^{a} u^{b}+p g^{a b}+\pi^{a b}
$$


The quantity $\pi^{a b}=\sqrt{3} S(r)\left(c^{a} c^{b}-\frac{1}{3} h^{a b}\right)$ is the anisotropic stress tensor; the spacelike vector $c^{a}=e^{-\lambda / 2} \delta_{1}^{a}$ is orthogonal to the fluid four-velocity $u^{a}=e^{-\nu / 2} \delta_{0}^{a}$ and $|S(r)|$ is the magnitude of the stress tensor. The Einstein field equations, with (11) and (15), can be written as

$$
\begin{aligned}
e^{-\lambda} & =1-\frac{2 m}{r} \\
r(r-2 m) \nu^{\prime} & =p_{r} r^{3}+2 m \\
\left(\mu+p_{r}\right) \nu^{\prime}+2 p_{r}^{\prime} & =-\frac{4}{r}\left(p_{r}-p_{\perp}\right)
\end{aligned}
$$

for anisotropic matter distributions. The quantities $p_{r}$ and $p_{\perp}$ are the radial and tangential pressures respectively. The radial pressure $p_{r}=p+2 S / \sqrt{3}$ and the tangential pressure $p_{\perp}=p-S / \sqrt{3}$ are not equal for anisotropic matter. Note that for isotropic matter $S=0$ and $p_{r}=p_{\perp}=p$ and we regain (4). The magnitude $S$ provides a measure of anisotropy.

\section{The Algorithm}

In this section we establish a procedure for generating a new anisotropic solution of the Einstein field equations from a known isotropic solution. Consider the Einstein field equations (4) with isotropic matter distribution. Suppose an explicit solution to (4) is known where

$$
(\nu, \lambda, m, p)=\left(\nu_{0}, \lambda_{0}, m_{0}, p_{0}\right)
$$


and the functions $\nu_{0}, \lambda_{0}, m_{0}$ and $p_{0}$ are explicitly given. Then the equations in (41) are satisfied and we can write

$$
\begin{aligned}
e^{-\lambda_{0}} & =1-\frac{2 m_{0}}{r} \\
r\left(r-2 m_{0}\right) \nu_{0}^{\prime} & =p_{0} r^{3}+2 m_{0} \\
\left(\frac{2 m_{0}^{\prime}}{r^{2}}+p_{0}\right) \nu_{0}^{\prime}+2 p_{0}^{\prime} & =0
\end{aligned}
$$

The equations in (8) correspond to an isotropic relativistic sphere. Now consider the Einstein field equations (6) with anisotropic matter distribution. We seek an explicit solution to the system (6). To this end we propose the possible solution

$$
\left(\nu, \lambda, m, p_{r}, p_{\perp}\right)=\left(\nu_{0}+\beta(r), \lambda_{0}, m_{0}, p_{0}+\alpha(r), p_{0}-\frac{1}{2} \alpha(r)\right)
$$

where $\left(\nu_{0}, \lambda_{0}, m_{0}, p_{0}\right)$ are given by (17), $\alpha$ and $\beta$ are arbitrary functions, and we have set $\alpha=2 S / \sqrt{3}$ for convenience. The equations (91) apply to the anisotropic relativistic sphere.

With the specified form (9), the system (6) becomes

$$
\begin{aligned}
e^{-\lambda_{0}} & =1-\frac{2 m_{0}}{r} \\
r\left(r-2 m_{0}\right) \nu_{0}^{\prime}+r\left(r-2 m_{0}\right) \beta^{\prime} & =p_{0} r^{3}+\alpha r^{3}+2 m_{0} \\
\left(\frac{2 m_{0}^{\prime}}{r^{2}}+p_{0}+\alpha\right)\left(\nu_{0}^{\prime}+\beta^{\prime}\right)+2 p_{0}^{\prime}+2 \alpha^{\prime} & =-\frac{6}{r} \alpha
\end{aligned}
$$

The systems (8) and (10) imply that

$$
\begin{aligned}
\left(r-2 m_{0}\right) \beta^{\prime} & =\alpha r^{2} \\
\left(\frac{2 m_{0}^{\prime}}{r^{2}}+p_{0}\right) \beta^{\prime}+\alpha\left(\nu_{0}^{\prime}+\beta^{\prime}\right)+2 \alpha^{\prime} & =-\frac{6}{r} \alpha
\end{aligned}
$$

which is a coupled system of first order equations. It remains to integrate (11a) and obtain $\alpha$ and $\beta$. 
We can write (11b) as

$$
\left(\frac{2 m_{0}^{\prime}}{r^{2}}+p_{0}\right) \frac{r^{2}}{r-2 m_{0}}+\nu_{0}^{\prime}+\beta^{\prime}+2 \frac{\alpha^{\prime}}{\alpha}=-\frac{6}{r}
$$

with the help of (11a). This differential equation can be integrated to give

$$
\alpha=\frac{k}{r^{3}} \exp \left\{-\frac{1}{2}\left(I_{\alpha}+\nu_{0}+\beta\right)\right\}
$$

where $k$ is a constant, and we have set

$$
I_{\alpha}=\int\left(\frac{2 m_{0}^{\prime}}{r^{2}}+p_{0}\right) \frac{r^{2}}{r-2 m_{0}} \mathrm{~d} r
$$

From (11a) and (12) we generate the nonlinear differential equation in $\beta$ :

$$
e^{\beta / 2} \beta^{\prime}=\frac{k}{r\left(r-2 m_{0}\right)} \exp \left\{-\frac{1}{2}\left(I_{\alpha}+\nu_{0}\right)\right\}
$$

This nonlinear first order differential equation is integrable and we generate the result

$$
\beta=2 \ln \left\{\frac{k}{2} I_{\beta}+\ell\right\}
$$

where $\ell$ is a constant of integration, and we have set

$$
I_{\beta}=\int \frac{\exp \left\{-\frac{1}{2}\left(I_{\alpha}+\nu_{0}\right)\right\}}{r\left(r-2 m_{0}\right)} \mathrm{d} r
$$

Thus the anisotropic field equations (6) have been integrated to generate a new exact solution. Note that with $k=0(=\alpha)$ this algorithm regains the isotropic solution (17). When $k \neq 0$ then the solution is necessarily anisotropic. The integrations in $I_{\alpha}$ and $I_{\beta}$ in (13) and (15) respectively can be performed explicitly as $\nu_{0}, p_{0}$ and $m_{0}$ are specified in the isotropic solution functions in (17). We make two observations relating to the constants of integration. These could have been introduced at a later stage as the solution is expressed in terms of indefinite integrals. If $k=0$ then the parameter $\ell$ has to be greater than zero and can be removed by rescaling the time coordinate. 
We summarise our result in terms of the following theorem:

Theorem $\mathcal{A}$ : If $\left(\nu_{0}, \lambda_{0}, m_{0}, p_{0}\right)$ is a given isotropic solution of the Einstein field equations then $\left(\nu, \lambda, m, p_{r}, p_{\perp}\right)=\left(\nu_{0}+\beta(r), \lambda_{0}, m_{0}, p_{0}+\alpha(r), p_{0}-\frac{1}{2} \alpha(r)\right)$ is a new anisotropic solution where

$$
\begin{aligned}
& \alpha=\frac{k}{r^{3}} \exp \left\{-\frac{1}{2}\left(\int\left[\frac{2 m_{0}^{\prime}}{r^{2}}+p_{0}\right] \frac{r^{2}}{r-2 m_{0}} \mathrm{~d} r+\nu_{0}+\beta\right)\right\} \\
& \beta=2 \ln \left\{\frac{k}{2} \int \frac{\exp \left\{-\frac{1}{2}\left(\int\left[\frac{2 m_{0}^{\prime}}{r^{2}}+p_{0}\right] \frac{r^{2}}{r-2 m_{0}} \mathrm{~d} r+\nu_{0}\right)\right\}}{r\left(r-2 m_{0}\right)} \mathrm{d} r+\ell\right\}
\end{aligned}
$$

and $k$ and $\ell$ are constants.

\section{Anisotropic isothermal spheres}

As a first example we illustrate the applicability of Theorem $\mathcal{A}$ by generating anisotropic isothermal spheres. The line element for the isothermal model [13] is

$$
\mathrm{d} s^{2}=-r^{\frac{4 c}{1+c}} \mathrm{~d} t^{2}+\left(1+\frac{4 c}{(1+c)^{2}}\right) \mathrm{d} r^{2}+r^{2}\left(\mathrm{~d} \theta^{2}+\sin ^{2} \theta \mathrm{d} \phi^{2}\right)
$$

where $c$ is a constant. The relevant isotropic functions for (16) are

$$
\begin{aligned}
\left(\nu_{0}, \lambda_{0}, m_{0}, p_{0}\right)= & \left(\frac{4 c}{1+c} \ln r, \ln \left\{1+\frac{4 c}{(1+c)^{2}}\right\},\right. \\
& \left.\frac{2 c r}{4 c+(1+c)^{2}}, \frac{1}{r^{2}} \frac{4 c^{2}}{4 c+(1+c)^{2}}\right)
\end{aligned}
$$

The energy density function, that generates the mass function $m_{0}$, has the form

$$
\mu_{0}=\frac{4 c}{4 c+(1+c)^{2}} \frac{1}{r^{2}}
$$


From (18) and $p_{0}$ in (17) we observe that

$$
p_{0}=c \mu_{0}
$$

which is a linear barotropic equation of state. Isothermal spheres with $\mu \propto$ $r^{-2}$ and the equation of state (19) arise in both Newtonian and relativistic stars [11, 12]. They have a long history in astrophysics as an equilibrium approximation to more complicated systems which are close to a dynamically relaxed state [14].

With the isotropic functions (17) it is possible to evaluate the integrals $I_{\alpha}$ and $I_{\beta}$ in (13) and (15). Then we generate the expressions

$$
\begin{aligned}
& \alpha=k\left(\ell-\frac{k}{2} \frac{4 c+(1+c)^{2}}{(1+c)(1+5 c)} r^{-\frac{1+5 c}{1+c}}\right)^{-1} r^{-\frac{3+7 c}{1+c}} \\
& \beta=2 \ln \left\{\ell-\frac{k}{2} \frac{4 c+(1+c)^{2}}{(1+c)(1+5 c)} r^{-\frac{1+5 c}{1+c}}\right\}
\end{aligned}
$$

Hence the new line element has the form

$$
\begin{aligned}
\mathrm{d} s^{2}= & -r^{\frac{4 c}{1+c}}\left(\ell-\frac{k}{2} \frac{4 c+(1+c)^{2}}{(1+c)(1+5 c)} r^{-\frac{1+5 c}{1+c}}\right)^{2} \mathrm{~d} t^{2}+\left(1+\frac{4 c}{(1+c)^{2}}\right) \mathrm{d} r^{2} \\
& +r^{2}\left(\mathrm{~d} \theta^{2}+\sin ^{2} \theta \mathrm{d} \phi^{2}\right)
\end{aligned}
$$

and the matter variables have the explicit form

$$
\begin{aligned}
m= & \frac{2 c r}{4 c+(1+c)^{2}} \\
p_{r}= & \frac{1}{r^{2}} \frac{4 c^{2}}{4 c+(1+c)^{2}} \\
& +k\left(\ell-\frac{k}{2} \frac{4 c+(1+c)^{2}}{(1+c)(1+5 c)} r^{-\frac{1+5 c}{1+c}}\right)^{-1} r^{-\frac{3+7 c}{1+c}} \\
p_{\perp}= & \frac{1}{r^{2}} \frac{4 c^{2}}{4 c+(1+c)^{2}} \\
& -\frac{k}{2}\left(\ell-\frac{k}{2} \frac{4 c+(1+c)^{2}}{(1+c)(1+5 c)} r^{-\frac{1+5 c}{1+c}}\right)^{-1} r^{-\frac{3+7 c}{1+c}}
\end{aligned}
$$


The isotropic isothermal sphere model (16) generates the anisotropic isothermal sphere model (20). With the parameter values $k=0, \ell=1$, we regain the conventional isothermal sphere.

The degree of anisotropy is

$$
S=\frac{k}{2} \sqrt{3}\left(\ell-\frac{k}{2} \frac{4 c+(1+c)^{2}}{(1+c)(1+5 c)} r^{-\frac{1+5 c}{1+c}}\right)^{-1} r^{-\frac{3+7 c}{1+c}}
$$

A graph of the anisotropy factor (22) was plotted with the help of Mathematica 15]. This is given in Figure 1 for the particular values of the parameters shown. The anisotropy factor $S$ is plotted against the radial distance on the interval $0<r \leq 1$. It is worth noting from this graph that the anisotropy has the feature that it is a monotonically decreasing function as $r$ approaches the boundary subject to a particular choice of parameters. There is a singularity at $r=0$ which $S$ shares with the other dynamical and metric functions. However there are other choices of parameters that could be made such that $S$ is a monotonically increasing function if the physics of the problem demanded such behavior. An increasing profile for $S$ would be physically relevant for boson stars as pointed out by Dev and Gleiser [1]; however note that their analysis was performed for a constant energy density. The simple behavior of $S$ reflected in this graph indicates that a full physical investigation of this solution is possible; we will perform this investigation in future work.

\section{$5 \quad$ Anisotropic Schwarzschild spheres}

As a second example we illustrate the utility of Theorem $\mathcal{A}$ by generating anisotropic Schwarzschild spheres. The line element for the interior 


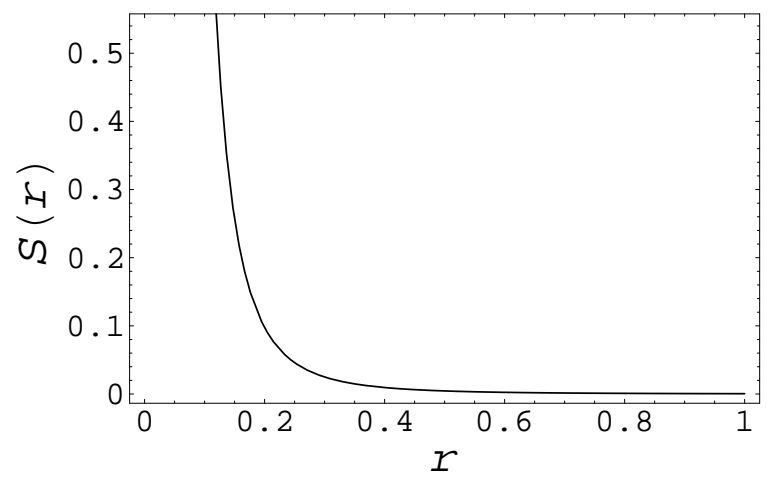

Figure 1: Decreasing $S(r)$ for anisotropic isothermal sphere; $c=0.1, k=$ 0.005 , and $\ell=10$

Schwarzschild model [16] is

$$
\begin{aligned}
\mathrm{d} s^{2}= & -\left(A-B \sqrt{1-\frac{r^{2}}{R^{2}}}\right)^{2} \mathrm{~d} t^{2}+\left(1-\frac{r^{2}}{R^{2}}\right)^{-1} \mathrm{~d} r^{2} \\
& +r^{2}\left(\mathrm{~d} \theta^{2}+\sin ^{2} \theta \mathrm{d} \phi^{2}\right)
\end{aligned}
$$

where $A$ and $B$ are constants. The relevant isotropic functions for (23) are

$$
\begin{aligned}
\left(\nu_{0}, \lambda_{0}, m_{0}, p_{0}\right)= & \left(2 \ln \left\{A-B \sqrt{1-\frac{r^{2}}{R^{2}}}\right\},-\ln \left\{1-\frac{r^{2}}{R^{2}}\right\},\right. \\
& \left.\frac{r^{3}}{2 R^{2}},-\frac{1}{R^{2}}\left(\frac{A-3 B \sqrt{1-\frac{r^{2}}{R^{2}}}}{A-B \sqrt{1-\frac{r^{2}}{R^{2}}}}\right)\right)
\end{aligned}
$$

The energy density function, that generates the mass function $m_{0}$, has the form $\mu_{0}=3 / R^{2}$. We therefore have

$$
\mu_{0}=\text { constant }
$$

which essentially replaces the equation of state (19) of Section 4 . The interior of dense neutron stars and superdense relativistic stars are of near uniform density [17, 18]. Consequently the assumption (25) of uniform energy density is often made in the modelling process [1, 9, 19]. 
The integral $I_{\alpha}$ in (13) is easily integrated and we obtain

$$
I_{\alpha}=2 \ln \left\{A-B \sqrt{1-\frac{r^{2}}{R^{2}}}\right\}-\ln \left\{1-\frac{r^{2}}{R^{2}}\right\}
$$

However the integral $I_{\beta}$ takes the form

$$
I_{\beta}=\int \frac{1}{r^{2} \sqrt{1-\frac{r^{2}}{R^{2}}}\left(A-B \sqrt{1-\frac{r^{2}}{R^{2}}}\right)^{2}} \mathrm{~d} r
$$

The integrand in (27) is complicated; however the integration can be completed and $I_{\beta}$ can be written purely in terms of elementary functions. The details of the integration are given in the Appendix.

We need to investigate two cases: $A \neq B$ and $A=B$. We observe from the Appendix that $I_{\alpha}$ and $I_{\beta}$ can be written in closed form for these two cases. Two line elements arise and we represent our solutions as follows:

Case $I: A \neq B$

In this case the line element is

$$
\begin{aligned}
\mathrm{d} s^{2}= & -\left(A-B \sqrt{1-\frac{r^{2}}{R^{2}}}\right)^{2} \\
& \times\left[\frac { k } { 2 R ( A ^ { 2 } - B ^ { 2 } ) ^ { 2 } } \left(\frac{6 A B^{2}}{\sqrt{B^{2}-A^{2}}} \tanh ^{-1}\left\{\frac{(A+B) \tan \left\{\frac{1}{2} \sin ^{-1} \frac{r}{R}\right\}}{\sqrt{B^{2}-A^{2}}}\right\}\right.\right. \\
& \left.\left.-\frac{R}{r}\left(2 A B+\left(A^{2}+B^{2}\right) \sqrt{1-\frac{r^{2}}{R^{2}}}\right)^{-1}\right)+\ell\right]^{2} \mathrm{~d} t^{2} \\
& \left.-\frac{B^{3} r}{R}\left(A-B \sqrt{1-\frac{r^{2}}{R^{2}}}\right)^{-1}\right) \\
& +\left(1-\frac{r^{2}}{R^{2}}\right)^{-1} \mathrm{~d} r^{2}+r^{2}\left(\mathrm{~d} \theta^{2}+\sin ^{2} \theta \mathrm{d} \phi^{2}\right)
\end{aligned}
$$


and the matter variables become

$$
\begin{aligned}
m= & \frac{r^{3}}{2 R^{2}} \\
p_{r}= & -\frac{1}{R^{2}}\left(\frac{A-3 B \sqrt{1-\frac{r^{2}}{R^{2}}}}{A-B \sqrt{1-\frac{r^{2}}{R^{2}}}}\right)+\frac{k \sqrt{1-\frac{r^{2}}{R^{2}}}}{r^{3}\left(A-B \sqrt{1-\frac{r^{2}}{R^{2}}}\right)^{2}} \\
& \times\left[\frac { k } { 2 R ( A ^ { 2 } - B ^ { 2 } ) ^ { 2 } } \left(\frac{6 A B^{2}}{\sqrt{B^{2}-A^{2}}} \tanh ^{-1}\left\{\frac{(A+B) \tan \left\{\frac{1}{2} \sin ^{-1} \frac{r}{R}\right\}}{\sqrt{B^{2}-A^{2}}}\right\}\right.\right. \\
& -\frac{R}{r}\left(2 A B+\left(A^{2}+B^{2}\right) \sqrt{1-\frac{r^{2}}{R^{2}}}\right) \\
& \left.\left.-\frac{B^{3} r}{R}\left(A-B \sqrt{1-\frac{r^{2}}{R^{2}}}\right)^{-1}\right)+\ell\right]^{-1} \\
p_{\perp}= & -\frac{1}{R^{2}}\left(\frac{A-3 B \sqrt{1-\frac{r^{2}}{R^{2}}}}{A-B \sqrt{1-\frac{r^{2}}{R^{2}}}}\right)^{-\frac{r^{2}}{R^{2}}} \\
& \times\left[\frac { k r ^ { 3 } ( A - B \sqrt { 1 - \frac { r ^ { 2 } } { R ^ { 2 } } } ) ^ { 2 } } { 2 R ( A ^ { 2 } - B ^ { 2 } ) ^ { 2 } } \left(\frac{6 A B^{2}}{\sqrt{B^{2}-A^{2}}} \tanh ^{-1}\left\{\frac{(A+B) \tan \left\{\frac{1}{2} \sin ^{-1} \frac{r}{R}\right\}}{\sqrt{B^{2}-A^{2}}}\right\}\right.\right. \\
& -\frac{R}{r}\left(2 A B+\left(A^{2}+B^{2}\right) \sqrt{1-\frac{r^{2}}{R^{2}}}\right) \\
& \left.\left.-\frac{B^{3} r}{R}\left(A-B \sqrt{1-\frac{r^{2}}{R^{2}}}\right)^{-1}\right)+\ell\right]^{-1}
\end{aligned}
$$

With the parameter values $k=0, \ell=1$ we regain the original interior Schwarzschild sphere (23). 
The degree of anisotropy is

$$
\begin{aligned}
S= & \frac{k \sqrt{3} \sqrt{1-\frac{r^{2}}{R^{2}}}}{2 r^{3}\left(A-B \sqrt{1-\frac{r^{2}}{R^{2}}}\right)^{2}} \\
& \times\left[\frac { k } { 2 R ( A ^ { 2 } - B ^ { 2 } ) ^ { 2 } } \left(\frac{6 A B^{2}}{\sqrt{B^{2}-A^{2}}} \tanh ^{-1}\left\{\frac{(A+B) \tan \left\{\frac{1}{2} \sin ^{-1} \frac{r}{R}\right\}}{\sqrt{B^{2}-A^{2}}}\right\}\right.\right. \\
& -\frac{R}{r}\left(2 A B+\left(A^{2}+B^{2}\right) \sqrt{1-\frac{r^{2}}{R^{2}}}\right) \\
& \left.\left.-\frac{B^{3} r}{R}\left(A-B \sqrt{1-\frac{r^{2}}{R^{2}}}\right)^{-1}\right)+\ell\right]^{-1}
\end{aligned}
$$

The graph of the anisotropy factor (30) was plotted with the assistance of Mathematica [15]. This is shown in Figure 2 for the particular values of the parameters. The interval for the plot of $S$ against $r$ is $0<r \leq 1$. The quantity $S$ is a monotonically decreasing function. Subject to the choice of the parameters, the anisotropy can be constructed such that it is a monotonically decreasing function as $r$ approaches the boundary. The function $S$ vanishes at the boundary. Other choices of the parameters $A, B, k$ and $\ell$ may generate different behavior for $S$. The singularity at the center does not seem to be 'removable' by any choice of parameters. A comparison of this case with Dev and Gleiser [1] is not possible as the anisotropy factor vanishes at the boundary $r=R$. This will be investigated further and a detailed analysis of the anisotropy factor $S$ and the dynamical variables for this anisotropic solution will be pursued in the future. 


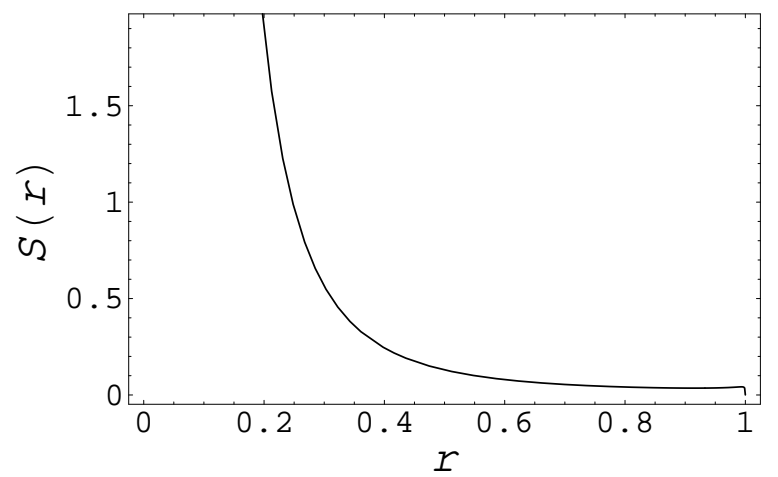

Figure 2: Decreasing $S(r)$ for anisotropic Schwarzschild sphere $(A \neq B)$; $A=1, B=-10, k=2, \ell=1$ and $R=1$.

Case II: $A=B$

For this case the line element is

$$
\begin{aligned}
\mathrm{d} s^{2}= & -\left(1-\sqrt{1-\frac{r^{2}}{R^{2}}}\right)^{2} \\
& \times\left[\ell-\frac{k}{80 R} \csc ^{5}\left\{\frac{1}{2} \sin ^{-1} \frac{r}{R}\right\} \sec \left\{\frac{1}{2} \sin ^{-1} \frac{r}{R}\right\}\right. \\
& \left.\times\left(\left(3-\frac{2 r^{2}}{R^{2}}\right) \sqrt{1-\frac{r^{2}}{R^{2}}}-2\left(1-\frac{2 r^{2}}{R^{2}}\right)\right)\right]^{2} \mathrm{~d} t^{2} \\
& +\left(1-\frac{r^{2}}{R^{2}}\right)^{-1} \mathrm{~d} r^{2}+r^{2}\left(\mathrm{~d} \theta^{2}+\sin ^{2} \theta \mathrm{d} \phi^{2}\right)
\end{aligned}
$$


and the matter variables are given by

$$
\begin{aligned}
& m=\frac{r^{3}}{2 R^{2}} \\
& p_{r}=-\frac{1}{R^{2}}\left(\frac{1-3 \sqrt{1-\frac{r^{2}}{R^{2}}}}{1-\sqrt{1-\frac{r^{2}}{R^{2}}}}\right) \\
& +\frac{k \sqrt{1-\frac{r^{2}}{R^{2}}}}{r^{3}\left(1-\sqrt{1-\frac{r^{2}}{R^{2}}}\right)^{2}}\left[\ell-\frac{k}{80 R} \csc ^{5}\left\{\frac{1}{2} \sin ^{-1} \frac{r}{R}\right\} \sec \left\{\frac{1}{2} \sin ^{-1} \frac{r}{R}\right\}\right. \\
& \left.\times\left(\left(3-\frac{2 r^{2}}{R^{2}}\right) \sqrt{1-\frac{r^{2}}{R^{2}}}-2\left(1-\frac{2 r^{2}}{R^{2}}\right)\right)\right]^{-1} \\
& p_{\perp}=-\frac{1}{R^{2}}\left(\frac{1-3 \sqrt{1-\frac{r^{2}}{R^{2}}}}{1-\sqrt{1-\frac{r^{2}}{R^{2}}}}\right) \\
& -\frac{k \sqrt{1-\frac{r^{2}}{R^{2}}}}{2 r^{3}\left(1-\sqrt{1-\frac{r^{2}}{R^{2}}}\right)^{2}}\left[\ell-\frac{k}{80 R} \csc ^{5}\left\{\frac{1}{2} \sin ^{-1} \frac{r}{R}\right\} \sec \left\{\frac{1}{2} \sin ^{-1} \frac{r}{R}\right\}\right. \\
& \left.\times\left(\left(3-\frac{2 r^{2}}{R^{2}}\right) \sqrt{1-\frac{r^{2}}{R^{2}}}-2\left(1-\frac{2 r^{2}}{R^{2}}\right)\right)\right]^{-1}
\end{aligned}
$$

With the parameter values $k=0, \ell=1$, we regain the original interior Schwarzschild sphere (23) with $A=B$.

The degree of anisotropy is

$$
\begin{aligned}
S= & \frac{k \sqrt{3} \sqrt{1-\frac{r^{2}}{R^{2}}}}{2 r^{3}\left(1-\sqrt{1-\frac{r^{2}}{R^{2}}}\right)^{2}}\left[\ell-\frac{k}{80 R} \csc ^{5}\left\{\frac{1}{2} \sin ^{-1} \frac{r}{R}\right\} \sec \left\{\frac{1}{2} \sin ^{-1} \frac{r}{R}\right\}\right. \\
& \left.\times\left(\left(3-\frac{2 r^{2}}{R^{2}}\right) \sqrt{1-\frac{r^{2}}{R^{2}}}-2\left(1-\frac{2 r^{2}}{R^{2}}\right)\right)\right]^{-1}
\end{aligned}
$$

The graph of the anisotropy factor (33) was plotted with the assistance of Mathematica [15]. This is shown in Figure 3 for the particular values of 
the parameters. The interval for the plot of $S$ against $r$ is $0<r \leq 1$. The quantity $S$ is a monotonically decreasing function. The behavior of $S$ is similar to the case $A \neq B$ given in Figure 2 However observe that the behavior in Figure 3 is more restricted for $C a s e I I$ as $A$ and $B$ are fixed.

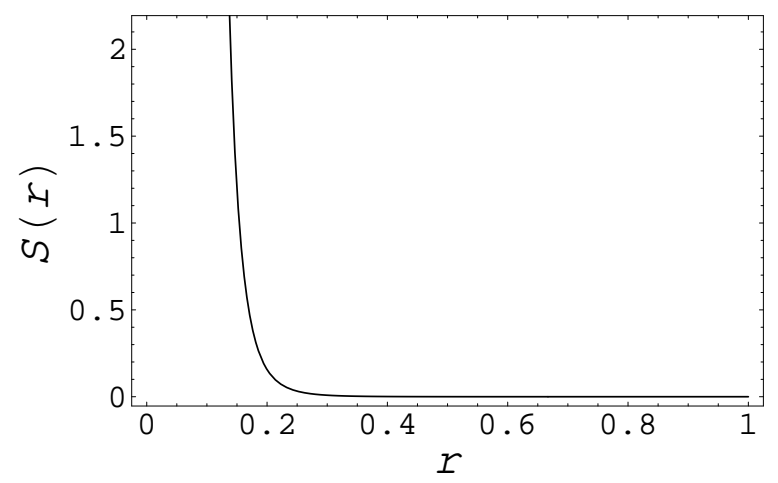

Figure 3: Decreasing $S(r)$ for anisotropic Schwarzschild sphere $(A=B)$; $k=0.00006, \ell=100$ and $R=1$

\section{Discussion}

It is remarkable that the integrations in Theorem $\mathcal{A}$ can be completed in closed form in spite of the complicated nature of the integrands in $\alpha$ and $\beta$, and we obtain expressions in terms of elementary functions. It is possible to demonstrate that the solutions found can be used to discuss the structure of neutron stars and quasi-stellar objects. Now consider a neutron star of radius 10 kilometres and surface density of $2 \times 10^{14} \mathrm{gcm}^{-3}$ in c.g.s units. Then the parameters relating to the surface redshift $z=(1-2 M / R)^{-1 / 2}-1$, and the mass $M$ in terms of the solar masses $M_{\odot}$ can be calculated. We choose values so that comparison with Gokhroo and Mehra [10] is facilitated. For the choice $c=0.1$, we obtain $z=0.154$ and $M=0.520 M_{\odot}$. The choice 
$c=0.9$, generates the values $z=0.413$ and $M=1.40 M_{\odot}$. Clearly other values for $z$ and $M$ can be generated by making different choices for $c$ and the surface density. The range of values obtainable is consistent with the results of Gokhroo and Mehra [10]. Hence our solutions yield values for surface redshifts and masses that correspond to realistic stellar sources such as Her X-1 and Vela X-1.

\section{Acknowledgements}

SDM and MC thank the National Research Foundation of South Africa for financial support. MC is grateful to the University of KwaZulu-Natal for a scholarship. We are grateful to the referees for valuable comments.

\section{Appendix}

The integral $I_{\beta}$ has the form

$$
I_{\beta}=\int \frac{1}{r^{2} \sqrt{1-\frac{r^{2}}{R^{2}}}\left(A-B \sqrt{1-\frac{r^{2}}{R^{2}}}\right)^{2}} \mathrm{~d} r
$$

Note that two cases arise in the integration process: $A \neq B$ and $A=B$.

Case $I: A \neq B$

To carry out the integration in (27) for $A \neq B$ we make the substitution

$$
\sin \vartheta=\frac{r}{R}
$$


so that $I_{\beta}$ becomes

$$
\begin{aligned}
I_{\beta}= & \frac{1}{R} \int \frac{1}{\sin ^{2} \vartheta(A-B \cos \vartheta)^{2}} \mathrm{~d} \vartheta \\
= & \frac{1}{R\left(A^{2}-B^{2}\right)^{2}}\left(\frac{6 A B^{2}}{\sqrt{B^{2}-A^{2}}} \tanh ^{-1}\left\{\frac{(A+B) \tan \frac{\vartheta}{2}}{\sqrt{B^{2}-A^{2}}}\right\}\right. \\
& \left.-\left(2 A B+A^{2} \cos \vartheta+B^{2} \cos \vartheta\right) \csc \vartheta-\frac{B^{3} \sin \vartheta}{A-B \cos \vartheta}\right)
\end{aligned}
$$

in terms of $\vartheta$. In terms of the original radial coordinate $r$ we have

$$
\begin{aligned}
I_{\beta}= & \frac{1}{R\left(A^{2}-B^{2}\right)^{2}}\left(\frac{6 A B^{2}}{\sqrt{B^{2}-A^{2}}} \tanh ^{-1}\left\{\frac{(A+B) \tan \left\{\frac{1}{2} \sin ^{-1} \frac{r}{R}\right\}}{\sqrt{B^{2}-A^{2}}}\right\}\right. \\
& \left.-\frac{R}{r}\left(2 A B+\left(A^{2}+B^{2}\right) \cos \left\{\sin ^{-1} \frac{r}{R}\right\}\right)-\frac{B^{3} r}{R\left(A-B \cos \left\{\sin ^{-1} \frac{r}{R}\right\}\right)}\right) \\
= & \frac{1}{R\left(A^{2}-B^{2}\right)^{2}}\left(\frac{6 A B^{2}}{\sqrt{B^{2}-A^{2}}} \tanh ^{-1}\left\{\frac{(A+B) \tan \left\{\frac{1}{2} \sin ^{-1} \frac{r}{R}\right\}}{\sqrt{B^{2}-A^{2}}}\right\}\right. \\
& \left.-\frac{R}{r}\left(2 A B+\left(A^{2}+B^{2}\right) \sqrt{1-\frac{r^{2}}{R^{2}}}\right)-\frac{B^{3} r}{R}\left(A-B \sqrt{1-\frac{r^{2}}{R^{2}}}\right)^{-1}\right)
\end{aligned}
$$

Then (12) and (14) give

$$
\begin{aligned}
\alpha= & \frac{k \sqrt{1-\frac{r^{2}}{R^{2}}}}{r^{3}\left(A-B \sqrt{1-\frac{r^{2}}{R^{2}}}\right)^{2}} \\
& \times\left[\frac { k } { 2 R ( A ^ { 2 } - B ^ { 2 } ) ^ { 2 } } \left(\frac{6 A B^{2}}{\sqrt{B^{2}-A^{2}}} \tanh ^{-1}\left\{\frac{(A+B) \tan \left\{\frac{1}{2} \sin ^{-1} \frac{r}{R}\right\}}{\sqrt{B^{2}-A^{2}}}\right\}\right.\right. \\
& -\frac{R}{r}\left(2 A B+\left(A^{2}+B^{2}\right) \sqrt{1-\frac{r^{2}}{R^{2}}}\right) \\
& \left.\left.-\frac{B^{3} r}{R}\left(A-B \sqrt{1-\frac{r^{2}}{R^{2}}}\right)^{-1}\right)+\ell\right]^{-1}
\end{aligned}
$$




$$
\begin{aligned}
\beta= & 2 \ln \left\{\frac { k } { 2 R ( A ^ { 2 } - B ^ { 2 } ) ^ { 2 } } \left(\frac{6 A B^{2}}{\sqrt{B^{2}-A^{2}}} \tanh ^{-1}\left\{\frac{(A+B) \tan \left\{\frac{1}{2} \sin ^{-1} \frac{r}{R}\right\}}{\sqrt{B^{2}-A^{2}}}\right\}\right.\right. \\
& -\frac{R}{r}\left(2 A B+\left(A^{2}+B^{2}\right) \sqrt{1-\frac{r^{2}}{R^{2}}}\right) \\
& \left.\left.-\frac{B^{3} r}{R}\left(A-B \sqrt{1-\frac{r^{2}}{R^{2}}}\right)^{-1}\right)+\ell\right\}
\end{aligned}
$$

for the functions $\alpha$ and $\beta$.

Case II: $A=B$

To carry out the integration in (27) with $A=B$ we again make the substitution

$$
\sin \vartheta=\frac{r}{R}
$$

to have

$$
\begin{aligned}
I_{\beta} & =\int \frac{1}{r^{2} \sqrt{1-\frac{r^{2}}{R^{2}}}\left(1-\sqrt{1-\frac{r^{2}}{R^{2}}}\right)^{2}} \mathrm{~d} r \\
& =\frac{1}{R} \int \frac{1}{\sin ^{2} \vartheta(1-\cos \vartheta)^{2}} \mathrm{~d} \vartheta \\
& =-\frac{1}{80 R} \csc ^{5} \frac{\vartheta}{2} \sec \frac{\vartheta}{2}(5 \cos \vartheta-4 \cos 2 \vartheta+\cos 3 \vartheta)
\end{aligned}
$$

The terms $\cos 2 \vartheta$ and $\cos 3 \vartheta$ can be simplified with basic trigonometric identities and $I_{\beta}$, in terms of the original radial coordinate $r$, becomes

$$
\begin{aligned}
I_{\beta}= & -\frac{1}{80 R} \csc ^{5}\left\{\frac{1}{2} \sin ^{-1} \frac{r}{R}\right\} \sec \left\{\frac{1}{2} \sin ^{-1} \frac{r}{R}\right\} \\
& \times\left(5 \sqrt{1-\frac{r^{2}}{R^{2}}}-4\left(1-\frac{r^{2}}{R^{2}}-\frac{r^{2}}{R^{2}}\right)+\sqrt{1-\frac{r^{2}}{R^{2}}}\left(1-\frac{r^{2}}{R^{2}}-3 \frac{r^{2}}{R^{2}}\right)\right) \\
= & -\frac{1}{80 R} \csc ^{5}\left\{\frac{1}{2} \sin ^{-1} \frac{r}{R}\right\} \sec \left\{\frac{1}{2} \sin ^{-1} \frac{r}{R}\right\} \\
& \times\left(\left(6-\frac{4 r^{2}}{R^{2}}\right) \sqrt{1-\frac{r^{2}}{R^{2}}}-4\left(1-\frac{2 r^{2}}{R^{2}}\right)\right)
\end{aligned}
$$


Then (12) and (14) give

$$
\begin{aligned}
\alpha= & \frac{k \sqrt{1-\frac{r^{2}}{R^{2}}}}{r^{3}\left(1-\sqrt{1-\frac{r^{2}}{R^{2}}}\right)^{2}}\left[\ell-\frac{k}{80 R} \csc ^{5}\left\{\frac{1}{2} \sin ^{-1} \frac{r}{R}\right\} \sec \left\{\frac{1}{2} \sin ^{-1} \frac{r}{R}\right\}\right. \\
& \left.\times\left(\left(3-\frac{2 r^{2}}{R^{2}}\right) \sqrt{1-\frac{r^{2}}{R^{2}}}-2\left(1-\frac{2 r^{2}}{R^{2}}\right)\right)\right]^{-1} \\
\beta= & 2 \ln \left\{\ell-\frac{k}{80 R} \csc ^{5}\left\{\frac{1}{2} \sin ^{-1} \frac{r}{R}\right\} \sec \left\{\frac{1}{2} \sin ^{-1} \frac{r}{R}\right\}\right. \\
& \left.\times\left(\left(3-\frac{2 r^{2}}{R^{2}}\right) \sqrt{1-\frac{r^{2}}{R^{2}}}-2\left(1-\frac{2 r^{2}}{R^{2}}\right)\right)\right\}
\end{aligned}
$$

for the functions $\alpha$ and $\beta$.

\section{References}

[1] Dev K, Gleiser M. Anisotropic stars: Exact solutions. General Relativity and Gravitation. 2002; 34: 1793-1818.

[2] Dev K, Gleiser M. Anisotropic stars II: Stability. General Relativity and Gravitation. 2003; 35: 1435-1457.

[3] Herrera L, Martin J, Ospino J. Anisotropic geodesic fluid spheres in general relativity. Journal of Mathematical Physics. 2002; 43: 4889-4897.

[4] Herrera L, Di Prisco A, Martin J, Ospino J, Santos NO, Troconis O. Spherically symmetric dissipative anisotropic fluids: A general study. Physical Review D. 2004; 69: 084026.

[5] Ivanov BV. Static charged perfect fluid spheres in general relativity. Physical Review D. 2002; 65: 104001. 
[6] Mak MK, Harko T. An exact anisotropic quark star model. Chinese Journal of Astronomy and Astrophysics. 2002; 2: 248-259.

[7] Mak MK, Harko T. Anisotropic stars in general relativity. Proceedings of the Royal Society A. 2003; 459: 393-408.

[8] Sharma R, Mukherjee S. Compact stars: A core-envelope model. Modern Physics Letters A. 2002; 17: 2535-2544.

[9] Maharaj SD, Maartens R. Anisotropic spheres with uniform energy density in general relativity. General Relativity and Gravitation. 1989; 21: 899-905.

[10] Gokhroo MK, Mehra AL. Anisotropic spheres with variable energy density in general relativity. General Relativity and Gravitation. 1994; 26: 75-84.

[11] Chaisi M, Maharaj SD. Compact anisotropic spheres with prescribed energy density. General Relativity and Gravitation. 2005; In press.

[12] Chaisi M, Maharaj SD. Anisotropic spheres in modelling highly compact bodies. Astrophysics and Space Science. 2005; Submitted.

[13] Saslaw WC, Maharaj SD, Dadhich N. An isothermal universe. Astrophysical Journal. 1996; 471, 571-574.

[14] Saslaw WC. Gravitational physics of stellar and galactic systems. Cambridge University Press: Cambridge, 1985.

[15] Wolfram S. Mathematica. Wolfram Research: Redwood City, 2003.

[16] Stephani S. Relativity: An introduction to special and general relativity. Cambridge University Press: Cambridge, 2004. 
[17] Maharaj SD, Leach PGL. Exact solutions for the Tikekar superdense star. Journal of Mathematical Physics. 1996; 37: 430-437.

[18] Rhoades CE, Ruffini R. Maximum mass of a neutron star. Physical Review Letters. 1974; 32: 324-327.

[19] Bowers RL, Liang EPT. Anisotropic spheres in general relativity. Astrophysical Journal. 1974; 188: 657-665. 